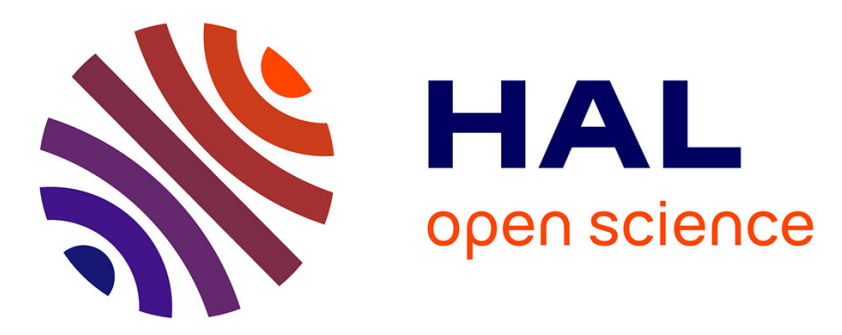

\title{
Evolutionary Changes of Pokemon Game: A Case Study with Focus On Catching Pokemon
}

Chetprayoon Panumate, Shuo Xiong, Hiroyuki Iida, Toshiaki Kondo

\section{To cite this version:}

Chetprayoon Panumate, Shuo Xiong, Hiroyuki Iida, Toshiaki Kondo. Evolutionary Changes of Pokemon Game: A Case Study with Focus On Catching Pokemon. 14th International Conference on Entertainment Computing (ICEC), Sep 2015, Trondheim, Norway. pp.182-194, 10.1007/978-3-31924589-8_14. hal-01758433

\section{HAL Id: hal-01758433 \\ https://hal.inria.fr/hal-01758433}

Submitted on 4 Apr 2018

HAL is a multi-disciplinary open access archive for the deposit and dissemination of scientific research documents, whether they are published or not. The documents may come from teaching and research institutions in France or abroad, or from public or private research centers.
L'archive ouverte pluridisciplinaire HAL, est destinée au dépôt et à la diffusion de documents scientifiques de niveau recherche, publiés ou non, émanant des établissements d'enseignement et de recherche français ou étrangers, des laboratoires publics ou privés. 


\title{
Evolutionary Changes of Pokemon Game: A Case Study with Focus On Catching Pokemon
}

\author{
Chetprayoon Panumate ${ }^{1}$ Shuo XIONG ${ }^{2}$ Hiroyuki Iida $^{3}$ and Toshiaki Kondo ${ }^{4}$ \\ 1 Japan Advanced Institute of Science and Technology, Japan \\ panumate.c@jaist.ac.jp $p^{1}$ \\ xiongshuo@jaist.ac.jp ${ }^{2}$ \\ iida@jaist.ac.jp $\mathrm{p}^{3}$ \\ 2 Sirindhorn International Institute of Technology, Thailand \\ tkondo@siit.tu.ac. th $^{4}$
}

\begin{abstract}
Game refinement is a unique theory that has been used as a reliable tool for measuring the attractiveness and sophistication of the games considered. A game refinement measure is derived from a game information progress model and has been applied in various games. In this paper, we aim to investigate the attractiveness of Pokemon, one of the most popular turn-based RPG games. We focus on catching Pokemons which are important components in the game. Then, we propose a new game refinement model with consideration on a prize cost and apply it to catching Pokemons. We analyze in every generation of the game. Experimental results show that a game refinement value of catching Pokemons which has been changed many times tries to reach to an appropriate range of game refinement value: $0.07-0.08$ for which previous works have confirmed.
\end{abstract}

Keywords: Game refinement theory, engagement, Pokemon

\section{Introduction}

Game theory [13] originated with the idea of the existence of mixed-strategy equilibrium in two-person zero sum games. It has been widely applied as a powerful tool in many fields such as economics, political science and computer science. Game refinement theory is another game theory focusing on attractiveness and sophistication of games based on the concept of information of game outcome uncertainty [7] [11]. The early works (e.g., [4] [8] [17] [20] [21]) focused on various types of games such as sports, board games and video games. The game refinement values of those popular games support the previous assumptions of a balanced range of game refinement value which is around $0.07-0.08$ [22] Classical game theory concerns the optimal strategy from the player's point of view, whereas game refinement theory concerns the optimization from the game designer's point of view.

In this study, we aim to investigate the attractiveness of Pokemon, one of the most popular turn-based RPG games [6]. While many efforts have been devoted 
to the study of Pokemon with focus on different points such as education [9], media science [15] and social science [6], the present study focuses on another important aspect of Pokemon: engagement or entertainment. Catching Pokemon is an important part that makes Pokemon game very attractive and widely popular.

For this study, we raise a research question: why has catching Pokemon mechanism been changed so many times in its history? To answer this question, we try to quantify the attractiveness of catching Pokemon in every episodes based on the game refinement theory. We propose a reasonable model of game information progress to derive a game refinement measure for catching Pokemon, while we consider prize cost to catch Pokemons.

In this paper we first give a short sketch of Pokemon game, especially for catching Pokemon. Then we present the fundamental idea of game refinement theory, and our new approach to the application of catching Pokemon with consideration on prize cost. Moreover, analyzed results obtained from various episodes are discussed and concluding remarks are given.

\section{Catching Pokemon}

In this section, we present a short history of Pokemon. Then, we focus on catching Pokemon which is our main target to apply game refinement model with consideration on prize cost. Moreover, some essential Pokemon capturing mechanism and equations are described.

\subsection{Pokemon}

Pokemon [2] [16] is a series of games developed by Game Freak and Creatures Inc. and published by Nintendo as part of the Pokemon media franchise. First released in 1996 in Japan for the Game Boy, the main series of role-playing video games (RPG) has continued on each generation of Nintendo's handhelds. Games are commonly released in pairs each with slight variations and then an enhanced remake of the games is released in a few years from the original release. While the main series consists of role-playing games, spinoffs encompass other genres such as action role-playing, puzzle and digital pet games. It is the second bestselling video game franchise worldwide, next to Nintendo's own Mario franchise. We show, in Table 1, a brief history of Pokemon.

The basic goal of Pokemon game [10] is to win the badges of gyms and become the champion of the league. For this purpose, one has to make his/her own team strong enough to win every battle in the game. Hence, in this study, we chiefly focus on the detail in catching Pokemon which is the main means to collect Pokemons and build one's own team.

\subsection{Catching Pokemon}

At the very initial stage to start a game, a Pokemon is given as a starter Pokemon for the coming adventure. The player may be able to catch other Pokemons by 
Table 1. History of Pokemon

\begin{tabular}{|c|c|c|c|}
\hline Generation & $\begin{array}{l}\text { Number of } \\
\text { Pokemons }\end{array}$ & Year & Version \\
\hline \multirow{3}{*}{1 st } & \multirow{3}{*}{151} & 1996 & Pokemon Red \& Green \\
\hline & & 1997 & Pokemon Blue \\
\hline & & 1998 & Pokemon Yellow \\
\hline \multirow{2}{*}{2 nd } & \multirow{2}{*}{251} & 1999 & Pokemon Gold \& Silver \\
\hline & & 2000 & Pokemon Crystal \\
\hline \multirow{3}{*}{$3 r d$} & \multirow{3}{*}{386} & 2002 & Pokemon Ruby \& Sapphire \\
\hline & & \multirow{2}{*}{2004} & Pokemon Fire Red \& Leaf Green \\
\hline & & & Pokemon Emerald \\
\hline \multirow{3}{*}{4 th } & \multirow{3}{*}{493} & 2006 & Pokemon Diamond \& Pearl \\
\hline & & 2008 & Pokemon Platinum \\
\hline & & 2009 & Pokemon Heart Gold \& Soul Silver \\
\hline \multirow{2}{*}{5 th } & \multirow{2}{*}{649} & 2010 & Pokemon Black \& White \\
\hline & & 2012 & Pokemon Black2 \& White2 \\
\hline \multirow{2}{*}{6 th } & \multirow{2}{*}{719} & 2013 & Pokemon X \& Y \\
\hline & & 2014 & Pokemon Omega Ruby \& Alpha Sapphire \\
\hline
\end{tabular}

his/her effort, except some Pokemons given automatically due to the story of the game. Importantly, the final goal of Pokemon game is to catch every Pokemon and make one's Pokedex, a portable device which provides information regarding the diversified species of Pokemon, be completed. Moreover, Table 1 shows that the number of Pokemons incessantly increases. We therefore understand that catching Pokemon is one of the most important parts in Pokemon game.

Catching Pokemon can be simply described that a player has to reduce the current HP of a target Pokemon as much as possible. HP [12] is an attribute assigned to each entity in game that indicates its state in combat. When HP of a player character reaches zero, the player may lose a life or their character might become incapacitated or die. So, to catch Pokemon, it is reasonable that the more Pokemon's HP is reduced, the weaker the target Pokemon is. Then, the player has to throw a ball, working as a catching device, to the Pokemon. Importantly, if the Pokemon is fainted, the catching attempt is unquestioningly failed. Additionally, using high quality ball or giving bad status to target Pokemon makes it easier to be caught.

According to Table 1, Pokemon has six generations to date. Each generation has own different catch rate mechanism except third generation and fourth generation, where these two generations follow the same mechanism. Below we show the detail for each catching mechanism [5].

$$
P_{1}=\frac{S}{B}+\frac{\min (C+1, B-S)}{B} \times \frac{\min (255, F)+1}{256}
$$

Where, $F=\left\lfloor\frac{\left\lfloor\frac{M \cdot 255}{G}\right\rfloor}{\max \left(1,\left\lfloor\frac{H}{4}\right\rfloor\right)}\right\rfloor$ 


$$
\begin{gathered}
P_{2}=\frac{\max \left(\frac{(3 M-2 H) \cdot C}{3 M}, 1\right)+S+1}{256} \\
P_{34}=\frac{\frac{(3 M-2 H) \cdot C B}{3 M} \cdot S}{255} \\
P_{5}=\frac{\frac{(3 M-2 H) \cdot G C B}{3 M} \cdot S \cdot \frac{E}{100}}{255} \\
P_{6}=\frac{\frac{(3 M-2 H) \cdot G C B}{3 M} \cdot S \cdot O}{255}
\end{gathered}
$$

$P_{i}$ stands for probability of catching Pokemon at $i$ th generation. $S$ is a variable for additional status. Normally, it is easiest to catch a Pokemon when its status is either asleep or frozen. The difficulty increases if the status is poisoned, burned, or paralyzed. The status none is the hardest case because it means that Pokemon is now very strong and ready to break any balls. $C$ is a capture rate. Every Pokemon has its own capture rate status between 3 and 255. The value 3 means that it is very hard to catch that Pokemon, it is for super rare or legendary Pokemon. The value 255 means that it is very easy to catch, it is for common Pokemon which can be found regularly. $B$ is a variable for ball used. There are many kinds of ball in this game. Some balls have special property which fit for some Pokemons whereas it also does not fit for other Pokemons. In this experiment, we focus on three kinds of common balls: Poke Ball, Great Ball and Ultra Ball, which can be bought in mart.

$G$ (for $P_{1}$ ) represents a variable for Great Ball modifier. Due to some bugs in the first generation, this variable makes Great Ball has a higher average catch rate than Ultra ball even though Ultra Ball is more expensive. $G$ (for $P_{5}$ and $P_{6}$ ) is a variable for grass modifier. It depends on the place where the player meets Pokemon. For example, if the action catching Pokemon takes place in thick grass, it is harder than normal grass. $E$ denotes Entralink power. During normal gameplay this value is not effective. However, by playing Entralink missions with their friends over local wireless, they can receive capturing power from another player, which enables them to increase the chance of catch rate. $O$ stands for $\mathrm{O}-$ Power bonus. This value replaces the Entralink modifier of the fifth-generation games to factor in Entralink powers sixth generation analogue, O-Powers.

$M$ means maximum HP. It can be exactly calculated by Equation (6) for first and second generation, and by Equation (7) for third generation onward. $H$ stands for current HP. It is reasonable that the more Pokemon's HP is reduced, the easier target Pokemon is caught. Importantly, if one makes the target Pokemon fainted, the catching attempt is unquestioningly failed.

$$
H P_{12}=\frac{\left(I V+\text { BaseHP }+\frac{E V}{8}+50\right) \times \text { Level }}{50}+10
$$




$$
H P_{3456}=\frac{\left(I V+2 \cdot \text { BaseHP }+\frac{E V}{4}+100\right) \times \text { Level }}{100}+10
$$

$I V$ is an individual value which is randomly generated by the game at the time when one meets that Pokemon first. There are six $I V$ due to each Pokemon has six battle status. To calculate HP, $I V$ of HP is used. BaseHP means initial HP status of Pokemon considered. It depends on what kind of one's Pokemon is. Some Pokemons have outstanding BaseHP while other Pokemons have poor BaseHP. Rationally, Pokemon with poor BaseHP should have another great initial status. However, legendary Pokemon may have excellent value in every initial status. $E V$ stands for special value which Pokemon will receive when finishing a battle. It depends on what kind of Pokemon has been defeated. Some Pokemons give $E V$ of HP while other Pokemons give $E V$ of another status. Level denotes a level of Pokemon considered. It simply starts from 1 to 100 . The more Pokemon's level increase, the stronger Pokemon becomes.

Moreover, there are various works which have been carried out with Pokemon, for example, in the domains of mathematics [1] and computer science [3]. In this paper, we focus on catching Pokemon which is an essential component in Pokemon game. Pokemon Catch Rate Calculator [5] is an application that enables us to calculate Pokemon catch rate in many situations and every generations. We use this tool for calculating the chance of catching Pokemon. In the next section, we show our new model, game refinement model with consideration on prize cost, which is applied to catching Pokemon.

\section{Assessment of Catching Pokemon}

In order to quantify the attractiveness of catching Pokemon, we first give a brief sketch of the basic idea of game refinement theory. Then we show our proposed model and its application to various episodes of Pokemon.

\subsection{Game refinement theory}

A general model of game refinement was proposed based on the concept of game progress and game information progress [17]. It bridges a gap between board games [8] and sports games [19]. We first show a general model of game progress in order to derive a game refinement measure. Then, we apply this idea to various games while identifying reasonable game progress models of given games, and compare them using game refinement measures.

The game progress is twofold [19]. One is game speed or scoring rate, while another one is game information progress with focus on the game outcome. Game information progress presents the degree of certainty of a game's result in time or in steps. Having full information of the game progress, i.e. after its conclusion, game progress $x(t)$ will be given as a linear function of time $t$ with $0 \leq t \leq t_{k}$ and $0 \leq x(t) \leq x\left(t_{k}\right)$, as shown in Equation (8). 


$$
x(t)=\frac{x\left(t_{k}\right)}{t_{k}} t
$$

However, the game information progress given by Equation (8) is unknown during the in-game period. The presence of uncertainty during the game, often until the final moments of a game, reasonably renders game progress as exponential. Hence, a realistic model of game information progress is given by Equation (9).

$$
x(t)=x\left(t_{k}\right)\left(\frac{t}{t_{k}}\right)^{n}
$$

Here $n$ stands for a constant parameter which is given based on the perspective of an observer of the game considered. Only a very boring game would progress in a linear function however, and most of course do not. Therefore, it is reasonable to assume a parameter $n$, based on the perception of game progress prior to completion. If the information of the game is completely known (i.e., after the end of the game) and the value of $n$ is 1 , the game progress curve appears as a straight line. In most games, especially in competitive ones, much of the information is incomplete, the value of $n$ cannot be assumed, and therefore game progress is a steep curve until its completion, along with $x\left(t_{k}\right), t_{k}, x(t)$ and $t$, just prior to game's end.

Then acceleration of game information progress is obtained by deriving Equation (9) twice. Solving it at $t=t_{k}$, we have Equation (10).

$$
x^{\prime \prime}\left(t_{k}\right)=\frac{x\left(t_{k}\right)}{\left(t_{k}\right)^{n}}\left(t_{k}\right)^{n-2} n(n-1)=\frac{x\left(t_{k}\right)}{\left(t_{k}\right)^{2}} n(n-1)
$$

It is assumed in the current model that game information progress in any type of game is encoded and transported in our brains. We do not yet know about the physics of information in the brain, but it is likely that the acceleration of information progress is subject to the forces and laws of physics. Too little game information acceleration may be easy for human observers and players to compute, and becomes boring. In contrast, too much game information acceleration surpasses the entertaining range and enters frustration, and at some point beyond that could become overwhelming and incomprehensible. Therefore we expect that the larger the value $\frac{x\left(t_{k}\right)}{\left(t_{k}\right)^{2}}$ is, the more the game becomes exciting, due in part to the uncertainty of game outcome. Thus, we use its root square, $\frac{\sqrt{x\left(t_{k}\right)}}{t_{k}}$, as a game refinement measure for the game under consideration. We call it $R$ value for short shown in Equation (11).

$$
R=\frac{\sqrt{x\left(t_{k}\right)}}{t_{k}}
$$

There are many works done before [14], the brief result is shown in Table 2 . From Table 2, we can propose suitable approach for various game types by using the described model. We see that sophisticated games have a $R$ value 
in the appropriate range, $0.07-0.08$. It has been proposed that information acceleration is one of the direct factors of engagement or excitement for all game types.

Table 2. Measures of game refinement for major board games and sports games

\begin{tabular}{|l|c|}
\hline Game & $R$ \\
\hline Chess & 0.074 \\
\hline Go & 0.076 \\
\hline Basketball & 0.073 \\
\hline Soccer & 0.073 \\
\hline
\end{tabular}

\subsection{Game refinement model with consideration on prizing cost}

While early work [18] focuses on playing cost, this work focuses on prizing cost. We propose $V$ as a value of each prize captured and $P$ as a probability of successful capturing the prize. Therefore, the game information $x\left(t_{k}\right)$ can be described as an average of $P$ and $V$, as given in Equation (12).

$$
x\left(t_{k}\right)=\frac{1}{n} \sum_{0<i<n} P_{i} V_{i}
$$

Next, we apply our $x\left(t_{k}\right)$ in game refinement measure $R$ in Equation (11). For this case, we have to calculate by percentage as shown in Equation (13).

$$
R=\frac{1}{10} \sqrt{\frac{1}{n} \sum_{0<i<n} P_{i} V_{i}}
$$

In order to apply this model to catching Pokemon, $V$, a value of each prize captured, can be calculated by the degree of rareness of target Pokemon. Normally, each Pokemon has its own capture rate which shows how hard to capture the target Pokemon, which means like a rareness of Pokemon. Therefore, we propose an equation for calculating the rareness of each Pokemon, $V$, as shown in Equation (14)

$$
V=\frac{M a x-C a p+M i n}{M a x-A v g}
$$

Max means the maximum of capture rate and Min means the minimum of capture rate. Likewise, $A v g$ means the average of capture rate and Cap means that target Pokemon's capture rate. Generally, the minimum of capture rate in Pokemon game is 3 , which means that it is very hard to catch. The maximum of capture rate in Pokemon game is 255 which means that it is very easy to catch this Pokemon. For the average of capture rate, it can be directly calculated which equals 100.25. Therefore, Equation (14) can be reduced in Equation (15). 


$$
V=\frac{256-C a p+3}{256-A v g}
$$

For probability of successful prize capturing, $P$, it can be calculated by Pokemon Catch Rate Calculator [5]. To use this calculator, we reasonably assume some parameters as explained below.

- Used Pokemon: We use 110 Pokemon samples which come from the first episode of Pokemon because we cannot use Pokemon from new episode to the first episode Pokemon Catch Rate Calculator. Moreover, the first episode of Pokemon contains 151 Pokemons but some Pokemon can be found from evolution. So we considerably cut some Pokemons which cannot be found as natural one.

- Current HP: We use 50 percent of full HP. It is the middle from 0 to 100.

- Pokemon level: It should be the average level of natural Pokemon in every episode which is approximately calculated by the lowest level and the maximum level of natural Pokemon in every episode. Finally, we already calculated average Pokemon level equals 25.69, approximately 26.

- Ball: There are many kinds of ball in Pokemon game. In this experiment, we focus on three main balls: Poke Ball, Great Ball and Ultra Ball.

- Status: Generally, if a Pokemon is asleep or frozen, it will be easiest to catch. If it is poisoned, burned or paralyzed, it is easier to catch but harder than asleep or frozen status. The hardest status for catching is none status. Hence, we use paralyzed status as an average status.

By this assumption, we obtain average of $P$ for each generation and ball used as shown in Table 3

Table 3. Average catch rate

\begin{tabular}{|l|c|c|c|}
\hline Generation & $R_{\text {PokeBall }}$ & $R_{\text {GreatBall }}$ & $R_{\text {UltraBall }}$ \\
\hline 1st & 39.14 & 63.98 & 52.55 \\
\hline 2nd & 33.36 & 42.60 & 47.86 \\
\hline 3rd \& 4th & 53.33 & 67.12 & 74.85 \\
\hline 5th & 57.03 & 69.54 & 76.50 \\
\hline 6th & 57.07 & 69.51 & 76.50 \\
\hline
\end{tabular}

Finally, we apply our new game refinement model with consideration on prize cost to Catching Pokemon. According to Equation (13), the result is shown in Table 4 .

\section{Discussion}

For our new game refinement model with consideration on prize cost as shown in Equation (13), we can consider it as a new approach to quantify attractiveness 
Table 4. Measures of game refinement for catching Pokemon with three main balls

\begin{tabular}{|l|c|c|c|}
\hline Generation & $R_{\text {PokeBall }}$ & $R_{\text {GreatBall }}$ & $R_{\text {UltraBall }}$ \\
\hline 1st & 0.047 & 0.062 & 0.059 \\
\hline 2nd & 0.042 & 0.050 & 0.056 \\
\hline 3rd \& 4th & 0.054 & 0.064 & 0.071 \\
\hline 5th & 0.058 & 0.067 & 0.072 \\
\hline 6th & 0.058 & 0.067 & 0.072 \\
\hline
\end{tabular}

of games. With this model, it enables us to explore new domains of game which cannot be investigated by previous models. The core of this model consists of two parameters, $P$ that stands for the probability of successful capturing and $V$ that stands for a value of each prize captured. For $P$, if we know the equation it can be calculated with the answer as the probability. If not, it can be calculated by simulating and collecting the data. For $V$, we have to carefully consider on what the related parameters of the prize's value are. Then, we establish the reasonable equation in order to measure the value of each prize.

Moreover, it has to be calculated in percentage system. We can apply this model to another game by creating reasonable equation. For the application to catching Pokemon, we collected data of catching Pokemon from Pokemon Catch Rate Calculator [5]. Then, we applied game refinement theory in the manner prescribed in Section 3.2. The results in Table 4 are compared in Figure (1).

For Poke Ball, we can see that in first generation the $R$ value is quite low. Furthermore, in second generation, the $R$ value is lower than first generation. Nevertheless, $R$ value is extremely increased in third generation and continuously go this way in fifth and sixth generation. Finally, it reaches 0.058 which is the maximum of $R$ value using Poke Ball in sixth generation.

For Great Ball, we can see that in first generation the $R$ value is not too low. However, in second generation, the $R$ value is awfully decreased from 0.062 in first generation to 0.50 . In third generation, $R$ comes back to 0.064 which is slightly higher than first generation. Then, it continuously increases and finally reach 0.067 in sixth generation.

For Ultra Ball, we can see that in first generation $R$ value is rather low. It then decreased from 0.059 in first generation to 0.056 in second generation. However, in third generation, $R$ significantly increased to 0.071 which is more than another $R$ value from another ball in another generation mentioned before. Then, it continuously increased to 0.072 in sixth generation which falls between the appropriate ranges of game refinement value: $0.07-0.08$.

Next, we consider each generation applied. According to Figure (1), in first generation, due to some errors, although Ultra ball is the best ball in these three kinds of balls but it has lower average catch rate and $R$ value than Great ball. That means in first generation some mechanism need to be fixed. In second generation, we can see that $R$ value for Ultra Ball is larger than Great Ball. That means the problem in previous generation was fixed. However, in second generation, every ball has trivially lower $R$ value than other generations. That means 


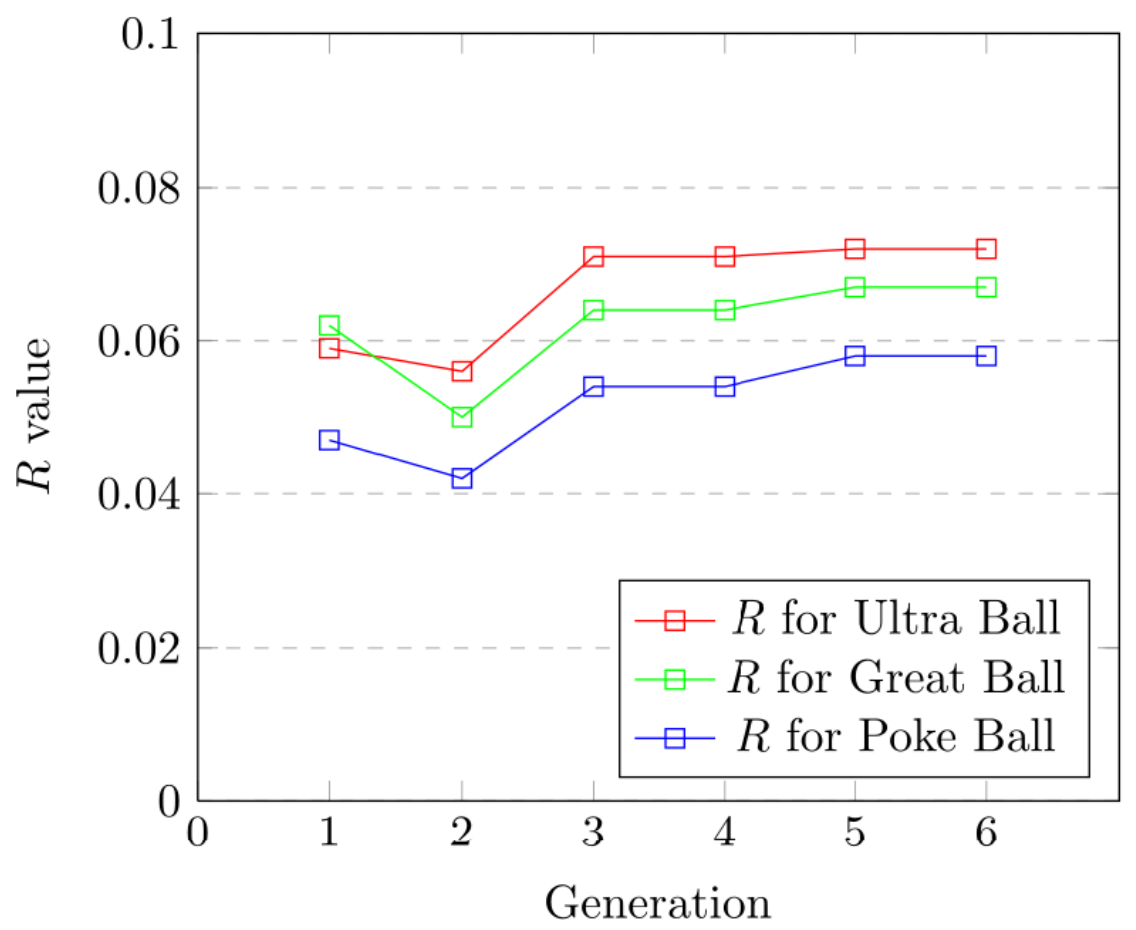

Fig. 1. Changes of game refinement values with three main balls compared 
the second generation's mechanism which is fixed from first generation does not work well and it should be fixed again. Suddenly, in third generation, $R$ value is remarkably developed and every ball in this generation has quite good $R$ value. Moreover, third generation catching mechanism is used in fourth generation which confirm the efficiency of this mechanism. For fifth and sixth generation, even though it uses the different catching mechanism but the calculated $R$ value is closely same. The calculated $R$ value of these mechanisms is very fine.

Moreover, we can consider the equation, both catching Pokemon equation and HP equation, directly. We see that the catching Pokemon mechanism which has been changed so many times try to add new parameters. For example, in fifth generation, Equation (4), proposing $E$ which is Entralink power. This enables players to receive a capture power from another player. This variable is changed to $O$ which is an O-Power bonus in sixth generation, Equation (5). We will see that both $E$ and $O$ increase a chance of capturing which may increase $R$ value.

To compare HP equation, we consider the modification from $H P_{12}$ to $H P_{3456}$ by reducing Equation (7) to Equation (16).

$$
H P_{3456}=\frac{\left(\frac{I V}{2}+\text { BaseHP }+\frac{E V}{8}+50\right) \times \frac{\text { Level }}{2}}{50}+10
$$

By comparing Equation (6) with Equation (16), we will see that BaseHP and $E V$ has the same coefficient while $I V$ and Level is changed. With this modification each Pokemon has lower HP value which trivially makes catching Pokemon easier.

Nevertheless, to get the more exact $R$ value, we should apply the idea of focusing on playing cost from early work [18]. In catching Pokemon, we can apply this idea by considering the cost of ball. We know that Poke Ball's cost is 200, Great Ball cost is 600 and Ultra Ball cost is 1200 . These costs have not been changed since the first generation of Pokemon until now. However, we cannot directly use the pure playing cost value because it will lead the $R$ value to be unreliable. We need to propose a reasonable function such as prize cost factor which was shown in Equation (13). To propose a reasonable function of playing cost, we need more data from other balls. Therefore, this work ignores this point and further works should carefully consider this issue.

Moreover, the experiments conducted in this study have many assumptions mentioned before. Further investigation may be made from difference aspects. Below we show a few examples.

- Used Pokemon: Normally, each episode will have its local Pokemon, a Pokemon that can be found in nature in that episode. We may consider each episode by those local Pokemons. So, each episode will use different Pokemon.

- Current HP: Current HP may be given randomly.

- Pokemon level: By following the used Pokemon assumption, we will consider each episode so that we can use each episode's average level of natural Pokemon as a Pokemon level in the experiment. So, each episode will use different Pokemon level. 
- Ball: We may focus on another ball.

- Status: We may assign a status for each Pokemon at random.

In conclusion, We can see that in first generation the result shows slightly lower values but it gradually increases in third generation and also in fifth and sixth generations. Finally, for Ultra Ball in third to sixth generation, the calculated $R$ value of catching Pokemon falls between $0.07-0.08$ which has been supposed to be a reliable game refinement value for many games that have undergone sophistication. The slightly lower value for the first and second generations implies that at that time the balance of catching mechanism, the relation between rareness of Pokemon and chance of catching was not optimized yet. Therefore, the catching mechanism was developed successively and it eventually reaches the appropriate range of $R$ value. However, the experiments in this study was performed with a simple model under many assumptions. Further works may try to focus on playing cost or use new assumptions.

\section{Conclusion}

We believe that catching Pokemons is an essential component of Pokemon game's engagement. This work is an attempt to find a reason why the mechanism for catching Pokemons has been changed so many times. To tackle this problem, we have applied a game refinement theory with a new model which considers a prize cost as shown in Equation (13). We have introduced a value of prize captured, $V$, because each prize has its own unique value. For the Pokemon case, we can calculate the value of captured prize by considering the rareness. We have proposed an equation for calculating the value $V$ from the rareness described in Equation (15). We used Pokemon Catch Rate Calculator, a reliable Pokemon catch rate simulator, as an essential tool in this research. The result is shown in Table 4 and is compared in Figure (1).

Our result confirms that the mechanism for catching Pokemons has been changed in a proper way by editing some details in the catch mechanism which directly increases $R$ value since the first generation to sixth generation. With the changing to an appropriate $R$ value, we conclude that this is the reason why the mechanism for catching Pokemon has been changed so many times. Moreover, we can predict that in the next generation, the catching mechanism will have $R$ value in an appropriate range.

It is obvious that game refinement theory can effectively be used in many domains of games such as classical board games, video games and sports, including Pokemon, by establishing a reasonable game information progress model. It can be used as a helpful tool to measure the attractiveness of a game and it also enables game designers to make a target game more sophisticated. As a conclusion, we have observed that a suitable game refinement value is around $0.07-0.08$, with many studies done before confirmation.

Additionally, the proposed model of game refinement which considers a prize cost can be applied in other domains where the value of each prize is unequal. We will be able to establish a reasonable equation to calculate a value of each 
prize. However, it is understood that the work presented in this study is a simple model with no complicated factors, and more studies are required. Further works may include to collect data in other types of ball or in other games which may have a catching component.

\section{Acknowledgement}

The authors wish to thank the anonymous referees for their constructive comments that helped to improve the article considerably. This research is funded by a grant from the Japan Society for the Promotion of Science, in the framework of the Grant-in-Aid for Challenging Exploratory Research (grant number 26540189).

\section{References}

1. G. Aloupis, E. D. Demainey, A. Guoyz and G. Vigliettax (2014). Classic Nintendo Games are (Computationally) Hard Fun with Algorithms-Lecture Notes in Computer Science Volume 8496, 2014, 40-51. Springer.

2. J. Bainbridge(2014) 'It is a Pokemon world': The Pokemon franchise and the environment International Journal of Cultural Studies July 2014 vol. 17 no. 4, 399-414.

3. S. D. Chen (2014). A Crude Analysis of Twitch Plays Pokemon arXiv preprint arXiv:1408.4925, 2014).

4. N. M. Diah, N. Nossal, N. A. M. Zin, T. Higuchi and H. Iida (2014). A Game Informatical Comparison of Chess and Association Football ("Soccer"), Advances in Computer Science 3(4), 10, 89-94

5. Dragonflycave pages, Pokemon Catch Rate Calculator. http://www.dragonflycave.com, URL accessed, 2015.

6. J. Horton (2012). Got my shoes, got my Pokemon: Everyday geographies of children popular culture. Geoforum Volume 43, Issue 1, January 2012, 413.

7. H. Iida, N. Takeshita, and J. Yoshimura. (2003). A metric for entertainment of boardgames: Its implication for evolution of chess variants. Entertainment Computing Technologies and Applications, 65-72.

8. H. Iida, K. Takahara, J. Nagashima, Y. Kajihara and T. Hashimoto. (2004). An application of game-refinement theory to Mah Jong. Entertainment ComputingICEC2004, 333-338. Springer.

9. Y. H. Lin (2007). Integrating Scenarios of Video Games into Classroom Instruction Information Technologies and Applications in Education, 200\%. ISITAE '0\%. First IEEE International Symposium on, 593-596, IEEE.

10. Y. H. Lin (2007). Pokemon: Game Play as Multi-Subject Learning Experience. The First IEEE International Workshop on Digital Game and Intelligent Toy Enhanced Learning (DIGITEL 'O7), 182-184.

11. P. Majek and H. Iida (2004). Uncertainty of Game Outcome, Proceedings of Inter Academia, 3rd Int. Conf. on Global Research and Education in Intelligent Systems. Budapest, Hungary, 71-180.

12. Moore, Michael (2011). Basics of Game Design. CRC Press. pp. 151-194. ISBN 1439867763.

13. J. Neumann. (1928). Zur theorie der gesellschaftsspiele. Mathematische Annalen, $100(1): 295-320$. 
14. N. Nossal and H. Iida (2014). Game Refinement Theory and Its Application to Score Limit Games. Proceedings of IEEE GEM 2014.

15. S. M. Ogletree, C. N. Martinez, T. R. Turner and B. Mason (2004). Pokemon: Exploring the Role of Gender Sex Roles June 2004, Volume 50, Issue 11-12, 851859. Springer.

16. Pokemon pages on wikipedia, http://www.wikipedia.com, URL accessed, 2015.

17. A. P. Sutiono, A. Purwarianti, and H. Iida. (2014). A mathematical model of game refinement, in D. Reidsma et al. (Eds.): INTETAIN 2014, LNICST 136, 148-151.

18. C. Rachaya, N. Nossal and H. Iida (2015). Game refinement model with consideration on playing cost: A case study using crane games. Knowledge and Smart Technology (KST), 2015 7th International Conference on, 87-92.

19. J. Takeuchi, R. Ramadan, and H. Iida. (2014). Game refinement theory and its application to Volleyball, Research Report 2014-GI-31(3), Information Processing Society of Japan, 1-6.

20. XIONG Shuo, ZUO Long and H. Iida (2014). Quantifying Engagement of Electronic Sports Game. Advances in Social and Behavioral Sciences, 5, 37-42.

21. XIONG Shuo and H.Iida (2014). Attractiveness of Real Time Strategy Games. International Conference on Systems and Informatics(ICSAI 2014), 264-269.

22. XIONG Shuo, ZUO Long, R. Chiewvanichakorn and H. Iida (2014). Quantifying Engagement of Various Games. The 19th Game Programming Workshop 2014, 101106. 